Journal of

Applied

Crystallography

ISSN 0021-8898

Editor: Anke R. Kaysser-Pyzalla

\title{
Crystalline perfection of an aluminium single crystal determined by neutron diffraction
}

\author{
Sabrina Metairon, Carlos Benedicto Ramos Parente, Vera Lucia Mazzocchi \\ and Thierry Jacques Lemaire
}

J. Appl. Cryst. (2011). 44, 681-687

Copyright (C) International Union of Crystallography

Author(s) of this paper may load this reprint on their own web site or institutional repository provided that this cover page is retained. Republication of this article or its storage in electronic databases other than as specified above is not permitted without prior permission in writing from the IUCr.

For further information see http://journals.iucr.org/services/authorrights.html

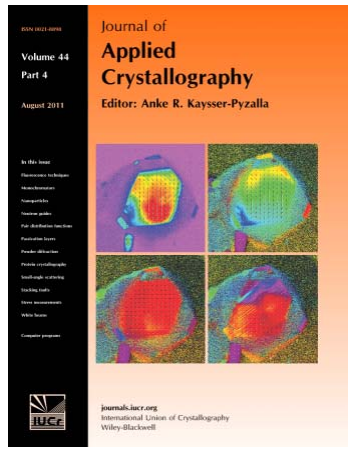

Journal of Applied Crystallography covers a wide range of crystallographic topics from the viewpoints of both techniques and theory. The journal presents papers on the application of crystallographic techniques and on the related apparatus and computer software. For many years, the Journal of Applied Crystallography has been the main vehicle for the publication of small-angle scattering papers and powder diffraction techniques. The journal is the primary place where crystallographic computer program information is published.

Crystallography Journals Online is available from journals.iucr.org 
Journal of

Applied

Crystallography

ISSN 0021-8898

Received 17 December 2010

Accepted 16 June 2011

\title{
Crystalline perfection of an aluminium single crystal determined by neutron diffraction
}

\author{
Sabrina Metairon, ${ }^{\mathrm{a}}$ Carlos Benedicto Ramos Parente, ${ }^{\mathrm{a} *}$ Vera Lucia Mazzocchi ${ }^{\mathrm{a}}$ and \\ Thierry Jacques Lemaire ${ }^{b}$
}

anstituto de Pesquisas Energéticas e Nucleares (IPEN-CNEN/SP), São Paulo, SP, Brazil, and

${ }^{\mathbf{b}}$ Universidade Federal da Bahia (UFBA), Salvador, BA, Brazil. Correspondence e-mail:

cparente@ipen.br

(C) 2011 International Union of Crystallography

Printed in Singapore - all rights reserved

\begin{abstract}
In this work, a study of the crystalline perfection of an aluminium single crystal is presented. The study shows that, from three-dimensional neutron diffraction rocking curves, it is possible to characterize the individual crystalline domains of a multidomain crystal. From a macroscopic point of view, the determination of the domains allows an evaluation of the crystalline perfection of the crystal under study. Three-dimensional rocking curves have been obtained by neutron diffraction from a large mosaic aluminium crystal. Construction of a contour map of individual domains made it easier to determine the breadth and relative intensity of each domain. The angular distances between domains were also determined.
\end{abstract}

\section{Introduction}

Baldochi et al. (1994) carried out a study of the crystalline quality of large Czochralski-grown barium lithium fluoride $\left(\mathrm{BaLiF}_{3}\right)$ single crystals. In that study, certain characteristics of neutron diffraction intensity curves (rocking curves) were correlated with several parameters involved in the growth method. As pointed out by the authors, neutrons are better than X-rays for this purpose since they give information about the bulk of the crystal. A neutron beam has, in general, a large cross-sectional area and a sample can be completely immersed in it. Furthermore, neutrons are highly penetrating particles for most materials. These two characteristics together make it easier to observe all crystalline domains of a single crystal, particularly if it is bulky. An X-ray beam, on the other hand, is much less penetrating than a neutron beam and has, in general, a small cross-sectional area. Thus, X-ray diffraction gives information about a very small surface portion of a large crystal. A single domain is then observed, unless the beam impinges upon a domain boundary. It is possible to use an $\mathrm{X}$-ray beam to observe many crystalline domains of a multidomain crystal, but a careful and exhaustive procedure must be adopted in order to sweep the entire surface of the crystal, which is undoubtedly very time consuming. Even then, domains located in the innermost part of the crystal will never be observed unless etching is used in the analysis.

Although the methodology employed in the study of Baldochi et al. (1994) led to useful results, it is not suitable for a complete characterization of the crystalline domains of a sample. This is due to the fact that the methodology used twodimensional instead of three-dimensional neutron diffraction rocking curves. In general, a two-dimensional rocking curve shows only a few crystalline domains; such domains appear as overlapping peaks. Except for those cases where the domains are closely aligned in the direction of the measurement, only a three-dimensional $I-\omega-\chi$ rocking curve can show all the domains of a crystal ( $I$ is the observed intensity of an $h \mathrm{kl}$ reflection, and $\omega$ and $\chi$ are two orthogonal rotations around axes that are both orthogonal to the scattering vector of the reflection). The domains are located at different regions in the $\omega-\chi$ grid. In principle, with a three-dimensional rocking curve it is possible to determine the number of domains, their relative intensities, the breadth of each one and the angular dispersion between them. From a macroscopic point of view, the determination of such characteristics allows an evaluation of the crystalline perfection of the crystal under study. The present work establishes a methodology for the analysis of the crystalline quality of single crystals using three-dimensional neutron diffraction rocking curves.

\section{Experimental}

A two-dimensional rocking curve can be obtained by turning the crystal around the $\omega$ axis of an Eulerian cradle while the intensity $I$ of an $h k l$ reflection is measured. During the measurement, the neutron detector remains in a fixed position at the scattering angle $2 \theta_{h k l}$ relative to the incident beam. This is the so-called moving-crystal-stationary-detector procedure. It corresponds to an $\omega$ scan in the normal-beam equatorial geometry (Arndt \& Willis, 1966). A three-dimensional $I-\omega-\chi$ rocking curve is formed using a set of two-dimensional $I-\omega$ rocking curves measured at different values of $\chi$, obtained by stepping this angle in convenient intervals. Fig. 1 is a schematic representation of the rotations used to obtain a threedimensional $I-\omega-\chi$ rocking curve.

To carry out the measurements, an experimental arrangement was assembled on the multipurpose neutron diffract- 
ometer installed at the IEA-R1 research reactor at IPEN. At the time of the measurements, the reactor was operating at a power of $2 \mathrm{MW}$ thermal. The 200 reflection of a large mosaic copper single crystal was used to obtain a monochromatic beam. At a take-off angle of $36^{\circ} 46^{\prime}$, the wavelength was $1.137 \AA$. The diffractometer was designed based on the normal-beam equatorial geometry. In the arrangement used for the rocking-curve measurements, the monochromatic beam Soller collimator was replaced with a special collimator which limits the vertical and horizontal divergences equally. This is the same collimator used to perform neutron multiple diffraction measurements on the instrument (Mazzocchi \& Parente, 1994, 1998). The beam cross section for this collimator is $3.8 \times 3.8 \mathrm{~cm}$ and the vertical and horizontal divergences are $c a 20^{\prime}$. The experimental arrangement also included a five-circle goniometer, which resembles an Eulerian cradle except that it has an extra horizontal axis $(\Sigma)$ appropriate for use in neutron multiple diffraction measurements (Mazzocchi \& Parente, 1994).

The crystal used in the measurements was a mosaic aluminium crystal shaped as a square plate, $7.6 \times 7.6 \times 1.3 \mathrm{~cm}$, and oriented with the (111) planes parallel to the square face. It was attached firmly to a goniometer head with the [111] direction approximately parallel to its central axis. The goniometer head was fastened to the $\varphi$ axis of the five-circle goniometer. This axis was rotated around the $\chi$ axis until it and the $\Sigma$ axis became coaxial. Careful orientation of the crystal in the neutron beam, in order to obtain the maximum intensity of the 111 reflection, preceded the measurements. The two-dimensional rocking curves were obtained by scanning $\omega$ in steps of $0.05^{\circ}$ with a count time of 2 min for each step. Although the area of the crystal surface was larger than the neutron beam cross section, the measurements were carried out without any sweep of the crystal in the beam. To obtain the three-dimensional rocking curve, the angle $\chi$ was varied in steps of $0.5^{\circ}$. Such a large step was possible since the $\chi$ scale is 'stretched' owing to the geometry of the measurements (see §3.4). Scans in both angular directions $\omega$ and $\chi$ were made large enough to extend the background for a few degrees on both sides of the curves.

To determine the instrumental broadenings for both $\omega$ and $\chi$ directions, we used a lithium fluoride (LiF) single crystal grown by the Czochralski method (Hagenmuller, 1972). Two two-dimensional 200 rocking curves were measured, one for each direction, $\omega$ or $\chi$. The rocking curve in the $\chi$ direction was measured after a $90^{\circ}$ rotation of the crystal around the $\varphi$

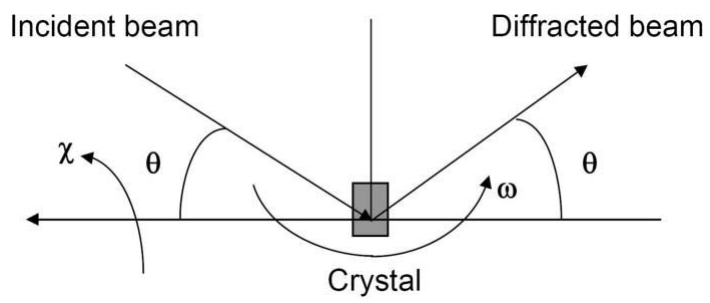

Figure 1

A schematic representation of the rotations $\omega$ and $\chi$ used to obtain a three-dimensional $I-\omega-\chi$ rocking curve. axis. This rotation was done in order to ensure that approximately the same region of the crystal used to obtain the instrumental broadening in the $\omega$ direction was also used to obtain the instrumental broadening in the $\chi$ direction. A careful Gaussian fit to both rocking curves showed that the crystal had several domains. The domain with the smallest values of the full widths at half-maximum (FWHMs) gave the values for the instrumental broadening. These are both represented by $\beta_{\mathrm{K}}$ and were 0.191 and $0.913^{\circ}$, respectively, for the $\omega$ and $\chi$ directions.

\section{Data treatment}

The purpose of the data treatment was to obtain the intrinsic three-dimensional crystalline domains of the aluminium crystal. For this purpose, each experimental two-dimensional rocking curve was fitted with Gaussians, followed by deconvolution of the Gaussians. Both processes were first applied to the rocking curves obtained in the $\omega$ direction and then to those obtained in the $\chi$ direction, the latter curves being extracted from the set of the former curves. An interpolation of points (Press et al., 1994) was performed prior to the deconvolution in order to guarantee better quality of the results. Contour maps were constructed with the deconvoluted data, allowing an easier characterization of the crystalline domains. All procedures employed in the data treatment are briefly described below.

\subsection{Deconvolution of Gaussians}

Deconvolution (or unfolding) of instrumental broadening from an experimental curve is, in general, a hard task with no guarantee of success. According to Press et al. (1994), apart from some mathematical problems, the process of deconvolution has other practical shortcomings. It is quite sensitive to noise in the input data and to the accuracy to which the response function is known. In this work, all such problems were overcome by simply assuming that the data must be fitted with Gaussian functions prior to the application of the deconvolution process. The process is then individually applied to each Gaussian fit. In this case, deconvolution becomes a straightforward mathematical process, without any additional difficulties. Clearly, this kind of solution is possible only because experimental neutron diffraction rocking curves can be fitted with Gaussian functions with excellent results.

A computer program was written in order to deconvolute the rocking curves. In a single run of the program, all Gaussians of a two-dimensional rocking curve can be deconvoluted. During the run, the deconvolution process is applied individually to each Gaussian according to the sequence in which they are listed in the input data. The output from the program gives the data for the deconvoluted rocking curve, as well as the parameters of each deconvoluted Gaussian. The program was first applied to all two-dimensional $I-\omega$ rocking curves, using the instrumental broadening in the $\omega$ direction. Twodimensional $I-\chi$ rocking curves were then extracted from the set formed by the deconvoluted $I-\omega$ rocking curves, one for 
each value of $\omega$ in its interval of variation. In a similar manner, using the instrumental broadening in the $\chi$ direction, $I-\chi$ rocking curves were deconvoluted. It should be noted that each interpolated point generates a new rocking curve in the transverse direction. This is valid for both $\omega$ and $\chi$ directions and causes a substantial increase in the number of rocking curves to be fitted and deconvoluted.

The three-dimensional rocking curve constructed from the raw experimental data obtained with the aluminium crystal is shown in Fig. 2(a). The three-dimensional rocking curve shown in Fig. 2(b) was constructed after interpolation and deconvolution of the two-dimensional rocking curves of Fig. 2(a), forming the deconvoluted three-dimensional $I-\omega-\chi$ rocking curve for the aluminium crystal. Interpolation of points is clearly seen when comparing the figures. An appreciable change in the two-dimensional rocking curves that form the surface enveloping the three-dimensional curve is also clearly seen.

\subsection{Fitting of data}

The fits were performed by means of a computer program that adjusts Gaussians to an experimental curve, using the least-squares method in the fitting process. It is not worthwhile to give a more detailed description of this program here. It belongs to a well known class of computer programs which perform the fitting of experimental curves with suitable mathematical functions. The program gives as output all three parameters of each Gaussian found in the fit, namely position, maximum intensity and FWHM. It also gives the area under each Gaussian, as well as the intensity points forming it. Parameters and areas are given with their standard deviations. An overall standard deviation for the fit itself is also given. A

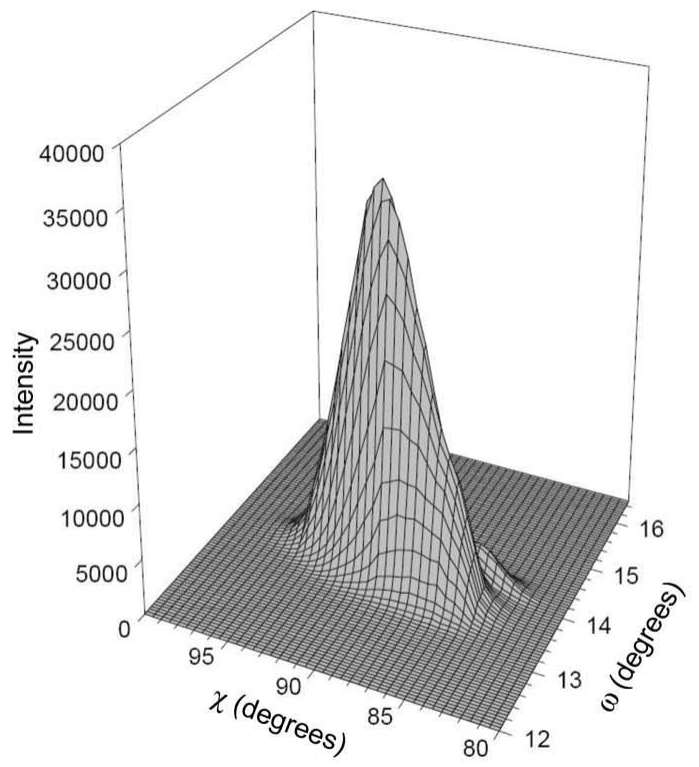

(a) serious drawback of this sort of program is that, in certain cases, the number of Gaussians to be specified for the fit becomes undefined. This happens when the curve to be fitted has several overlapping peaks with poor separation between them. This is the case in this work. A better description of the problem and its solution is given below.

During fitting of the numerous rocking curves we have verified that, after a certain number of Gaussians, the agreement between experimental and fitted curves experiences no substantial improvement. When the number of Gaussians becomes too large, the fit becomes erratic, although without spoiling the agreement. Figs. 3(a),3(b) and 3(c) are plots of the overall standard deviation $\sigma$ versus number of Gaussians $n$, obtained by fitting neighbouring rocking curves with, successively, $n=1,2,3,4$ or 5 Gaussians. They show how the agreement behaves with increasing $n$. Plots in the figures were obtained with $I-\omega$ rocking curves measured at, respectively, $\chi=$ $89.5,90$ and $90.5^{\circ}$. It is remarkable how $\sigma$ changes by two orders of magnitude when $n$ goes from 1 to 3 for all three rocking curves. From $n=3$ onwards $\sigma$ tends to stabilize. For this particular case, the number of Gaussians to be adopted should be at least $n=3$. However, a better choice of $n$ still depends on a qualitative evaluation of the fits, which should take into account how a Gaussian is altered when passing from one rocking curve to a neighbouring one. As a general rule for a non-deformed domain, the parameters of successive Gaussians, in particular the maximum intensity and FWHM, should change monotonically on both sides of the maximum. To ensure that the results of the fitting process are reliable, the fits must be checked continuously in order to verify if the domains follow the above behaviour. It is important to mention that, depending on the particular case, the procedure for the determination of $n$ must be applied again when domains

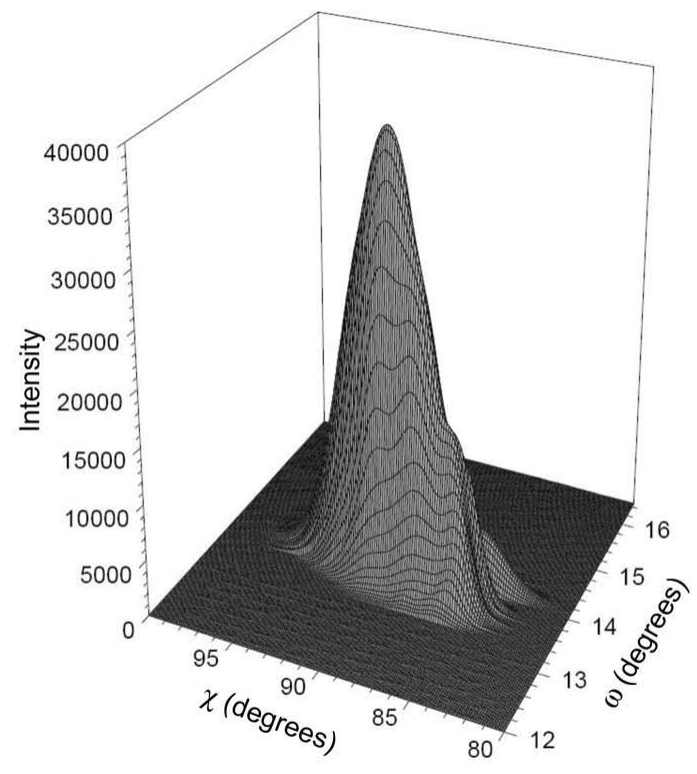

(b)

Figure 2

Three-dimensional $\mathrm{I}-\omega-\chi$ rocking curves constructed using $(a)$ the raw experimental data obtained from the aluminium crystal and $(b)$ after interpolation and deconvolution. 
appear or disappear in the sequence of rocking curves. As an illustration of the procedures described above, three Gaussians were chosen to initiate the fitting in the cases corresponding to Fig. 3.

\subsection{Contour maps}

A three-dimensional plot, like that of Fig. 2(b), does not allow a complete visualization of the rocking curve. Some details are always missing, unless the plot is rotated around a vertical axis and several plots are shown. For a better visualization of a three-dimensional rocking curve, a contour map must be constructed with the same data as used in the threedimensional plot.

\subsection{Reduction of the $\chi$ scale}

During determination of the instrumental broadening, we observed that the breadths of the rocking curves, measured in the directions $\omega$ and $\chi$, were very different. The breadth in the $\chi$ direction was $c a 16$ times that in the $\omega$ direction. Such a large difference could not be attributed to intrinsic characteristics of the crystal. In fact, as mentioned above, the crystal was rotated by $90^{\circ}$ for the measurement in the $\chi$ direction to ensure that essentially the same crystalline region was observed in both measurements. If not exactly the same, these regions were very alike in their characteristics. However, the enlargement of the rocking curve in the $\chi$ direction is in fact due to an enhancement of the Lorentz factor (Metairon, 1999). This

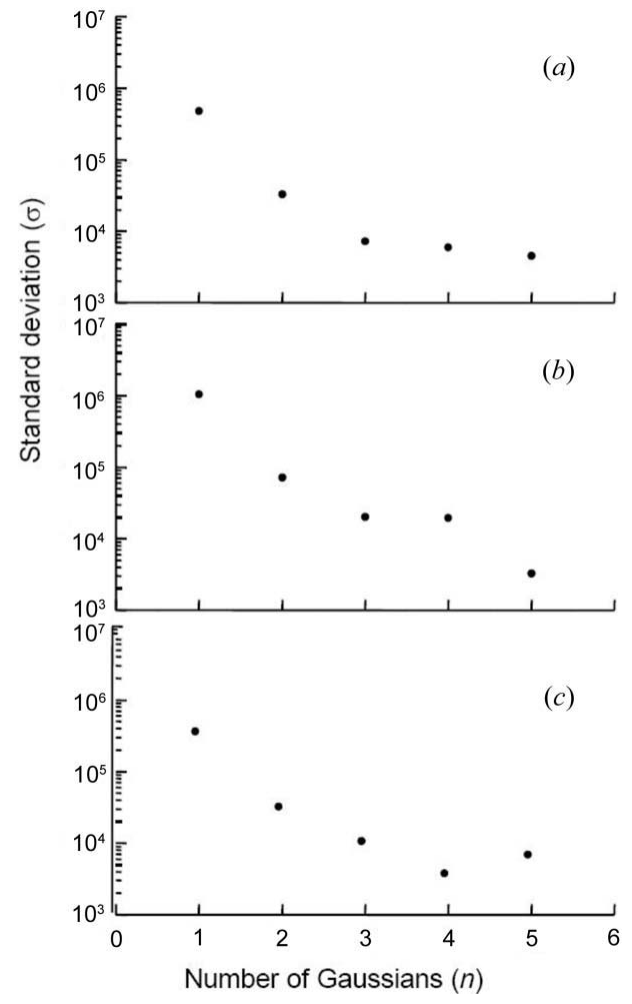

Figure 3

The overall standard deviation $(\sigma)$ versus number of Gaussians $(n)$ obtained by fitting $I-\omega$ rocking curves, measured at $(a) \chi=89.5^{\circ},(b) \chi=$ $90^{\circ}$ and $(c) \chi=90.5^{\circ}$, with one to five Gaussians. factor depends on the inclination, with respect to the surface of the Ewald sphere, of the trajectory described by a reciprocal lattice point during rocking of the crystal. The more inclined the trajectory, the more the rocking curve is enlarged. In an $\omega$ rocking of the crystal, the trajectory is almost perpendicular to the sphere and a small enlargement occurs. On the other hand, in a $\chi$ rocking the reciprocal lattice point approximates to the surface of the sphere at a grazing incidence and an enlarged rocking curve results (Rossmanith, 1986; Metairon, 1999). In order to reduce the enlargement in the $\chi$ direction a correction factor $f_{\text {corr }}$ was determined, which causes reduction of the $\chi$ scale. The reduction is such that the breadths of the twodimensional domains in one direction become of the same order of magnitude as those in the other direction. The correction factor was calculated by dividing the FWHM of a rocking curve measured in the $\omega$ direction by the FWHM of one measured in the $\chi$ direction, provided these rocking curves were measured in the same crystalline direction according to the procedure adopted in the determination of the instrumental broadening. The value of the correction factor for the aluminium crystal is $f_{\text {corr }}=0.0633$. Reduction of the $\chi$ scale is achieved by calculating the correct $\chi$ using the expression $\chi_{\text {corr }}=\left(\chi-\chi_{\text {max }}\right) f_{\text {corr }}$, where $\chi_{\max }$ is the $\chi$ coordinate of the point of maximum intensity in the threedimensional rocking curve.

Fig. 4 shows the contour map constructed for the aluminium crystal. In contrast with Figs. 2(a) and 2(b), Fig. 4 is plotted on a reduced $\chi$ scale where $\chi_{\max }=90^{\circ}$. The $\omega$ scale in the contour map is equal to that used in Fig. 2.

\subsection{Individual characterization of domains}

The contour map of Fig. 4 contains all the information required to allow an evaluation of the crystalline quality of the aluminium crystal. Nevertheless, it is not a useful source of information when individual characterization of the crystalline

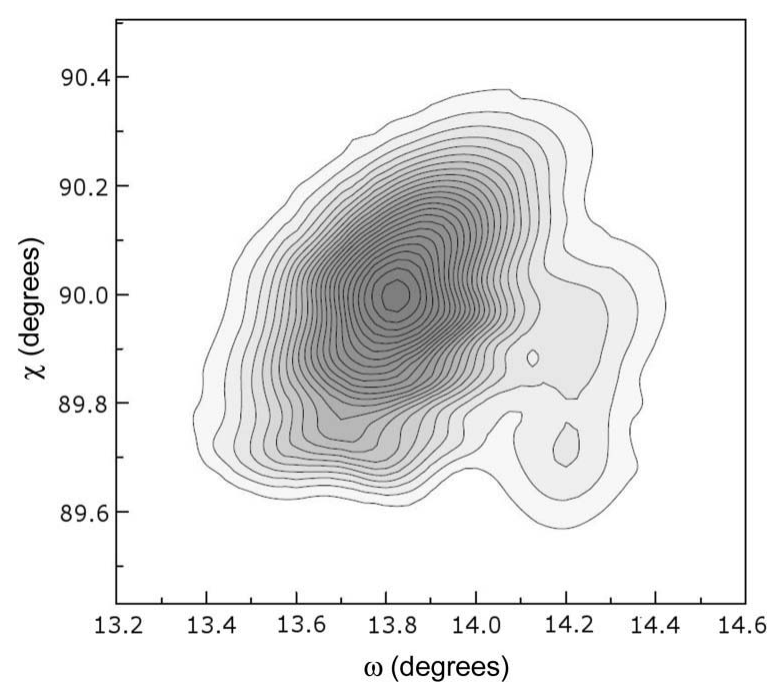

Figure 4

A contour map constructed for the aluminium crystal after deconvolution in both $\omega$ and $\chi$ directions. The $\chi$ scale was reduced using $\chi_{\max }=90^{\circ}$ and a correction factor $f_{\text {corr }}=0.0633$. 
domains and the relationships between them are sought. To achieve this objective, a contour map of the individual domains must be constructed. The construction is carried out using data collected from the outputs of the computer program used for the deconvolutions. It should be noted that a deconvoluted three-dimensional domain is formed via a certain number of deconvoluted Gaussians. Using the three parameters defining each such Gaussian, it is possible to individualize the domain in the contour map. It is worth mentioning that the direction assumed for the construction, $\omega$ or $\chi$, is irrelevant provided the same direction is used for the entire contour map. Extending this procedure to all domains of the crystal, a contour map of individual domains can be constructed. Fig. 5 shows a contour map of the individual domains constructed for the aluminium crystal. It combines the five three-dimensional domains found in the analysis of the crystal. Taking domain No. 3 (the highest one) as reference, the intensity levels assigned to the contour lines vary according to the following: the blank region on the map is zero, the first contour line corresponds to $0.3 \times 10^{4}$ counts, the second to $0.6 \times 10^{4}$, the third to $0.9 \times 10^{4}$, the fourth to $1.2 \times$ $10^{4}$ and so on. The eleventh and last contour line corresponds to $3.3 \times 10^{4}$ counts. To ascertain the intensity level of any other domain is a matter of finding the number of the contour line forming it.

Using the contour map of Fig. 5, the angular distances between the individual domains and their relative intensities and breadths can be determined. The procedures used to acquire this information and the results obtained are presented below.

\section{Results}

\subsection{Breadths of the domains}

The FWHM was assumed to be an adequate representation of the breadth of a domain. Since the domains are three-

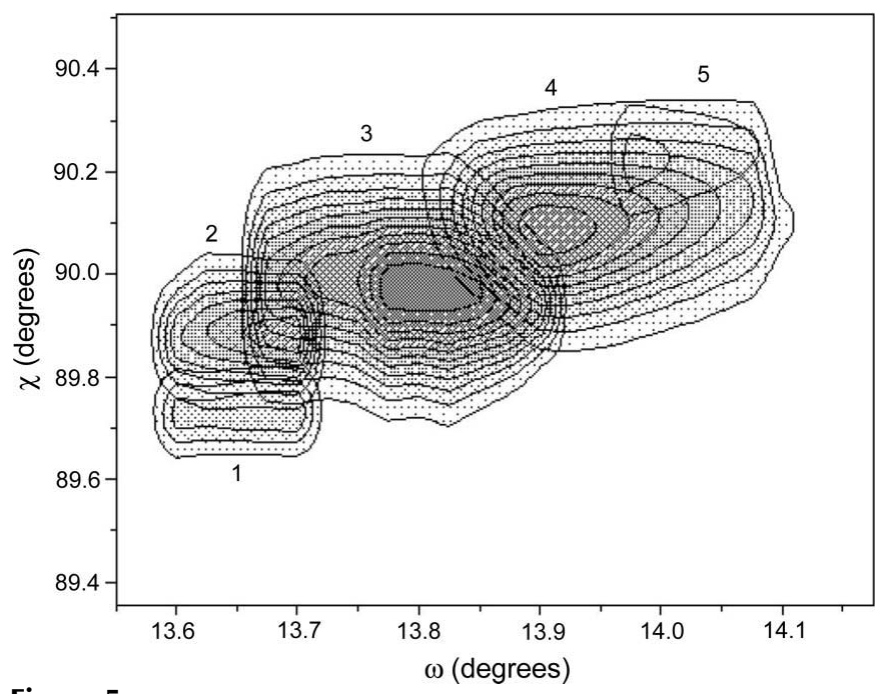

Figure 5

A contour map for the aluminium crystal, constructed with individual domains. dimensional, two values for the FWHM were considered, corresponding to directions $\omega$ and $\chi$. They are readily determined by first tracing orthogonal straight lines, intersecting at the point of maximum intensity of a domain. The lines are traced parallel to the $\omega$ and $\chi$ axes. The FWHMs in both directions are determined when the straight lines encounter the contour line corresponding to half of the maximum intensity.

Fig. 6 shows domain No. 3 of Fig. 5. In contrast with Fig. 5, the scale used to define the 11 contour lines in Fig. 6 is formed by the intensity levels 2982, 5965, 8947, 11 929, 14 911, 17 893, $20876,23858,25840,29822$ and 32805 counts. Using the values corresponding to the maximum intensity (35 787) and half of the maximum intensity (17 893), the FWHMs for directions $\omega$ and $\chi$ were determined following the procedure described above. They are indicated in Fig. 6 by $\beta_{\omega}$ and $\beta_{\chi}$ and their values listed in Table 1 , together with the values determined for the other domains. The corresponding values for the mosaic spreads $\eta_{\omega}$ and $\eta_{\chi}$ are also listed in Table 1.

\subsection{Angular distances between the domains}

In a contour map like that of Fig. 5, the angular distance separating two domains is represented by a line segment uniting the points of maximum intensity in the domains. Owing to the fact that $\omega$ and $\chi$ are angular scales, any line segment traced in the map is actually an arc of circle. Metairon (1999) observed that, for the determination of the angular distances, a spherical right triangle should be considered. In such a triangle, if $\Delta$ is the hypotenuse, i.e. the angular distance to be determined, $\Delta_{\omega}$ and $\Delta_{\chi}$ are the catheti, i.e. its components along directions $\omega$ and $\chi$, and $W\left(=90^{\circ}\right)$ is the opposite angle to arc $\Delta$, then according to the cosine law (Spiegel, 1968) the following relationships can be written:

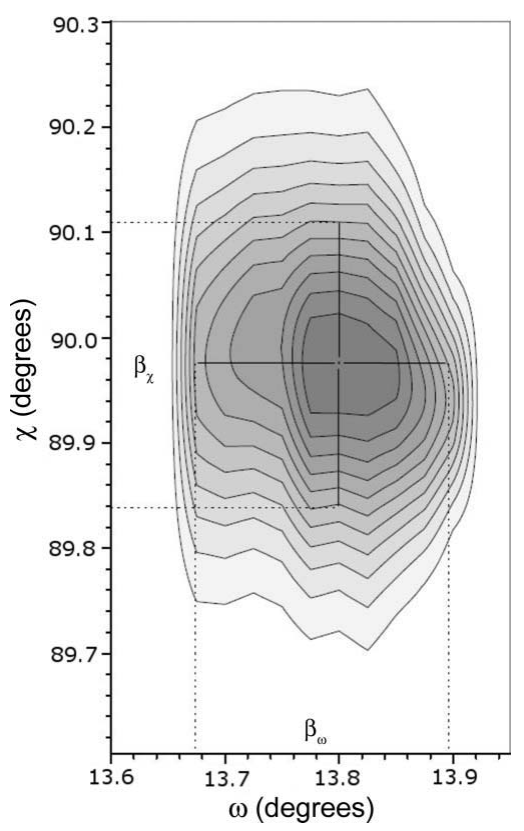

Figure 6

Determination of the FWHM of domain No. 3 of Fig. 5, for both $\omega$ and $\chi$ directions ( $\beta_{\omega}$ and $\beta_{\chi}$, respectively). 
Table 1

FWHM $(\beta)$ and standard deviation $(\eta)$ for both $\omega$ and $\chi$ directions, determined for the five crystalline domains of the aluminium crystal.

\begin{tabular}{lllll}
\hline Domain & $\beta_{\omega}\left(^{\circ}\right)$ & $\beta_{\chi}\left({ }^{\circ}\right)$ & $\eta_{\omega}\left(^{\circ}\right)$ & $\eta_{\chi}\left({ }^{\circ}\right)$ \\
\hline 1 & 0.123 & 0.125 & 0.052 & 0.053 \\
2 & 0.117 & 0.168 & 0.050 & 0.071 \\
3 & 0.224 & 0.269 & 0.095 & 0.114 \\
4 & 0.206 & 0.250 & 0.087 & 0.106 \\
5 & 0.095 & 0.176 & 0.040 & 0.075 \\
\hline
\end{tabular}

Table 2

Angular distances $(\Delta)$ between the five domains found in the aluminium crystal.

\begin{tabular}{llll}
\hline Domains & $\Delta_{\omega}\left({ }^{\circ}\right)$ & $\Delta_{\chi}\left(^{\circ}\right)$ & $\Delta\left(^{\circ}\right)$ \\
\hline 1 and 2 & 0 & 0.151 & 0.151 \\
1 and 3 & 0.100 & 0.246 & 0.266 \\
1 and 4 & 0.200 & 0.356 & 0.408 \\
1 and 5 & 0.275 & 0.491 & 0.563 \\
2 and 3 & 0.100 & 0.075 & 0.138 \\
2 and 4 & 0.200 & 0.205 & 0.286 \\
2 and 5 & 0.275 & 0.340 & 0.437 \\
3 and 4 & 0.100 & 0.110 & 0.149 \\
3 and 5 & 0.175 & 0.245 & 0.301 \\
4 and 5 & 0.075 & 0.350 & 0.154 \\
\hline
\end{tabular}

$$
\begin{aligned}
\cos \Delta & =\cos \Delta_{\omega} \cos \Delta_{\chi}+\sin \Delta_{\omega} \sin \Delta_{\chi} \cos W \\
& =\cos \Delta_{\omega} \cos \Delta_{\chi} .
\end{aligned}
$$

To calculate $\Delta$ between two domains, the points of maximum intensity in the domains must first be localized in the $\omega-\chi$ grid and then projected upon the axes $\omega$ and $\chi$. The angular lengths of the two line segments determined by the projections are then the values to be assigned to the rectangular components $\Delta_{\omega}$ and $\Delta_{\chi}$. With these two values, $\Delta$ can be easily calculated using the expression above. Table 2 lists the distances $\Delta$ calculated for the ten possible combinations between the domains of the aluminium crystal. The corresponding values for $\Delta_{\omega}$ and $\Delta_{\chi}$ are also listed in Table 2. As pointed out by Metairon (1999), $\Delta$ can also be calculated to a good approximation by assuming a planar right triangle and using the Pythagorean theorem, i.e. $\Delta^{2}=\Delta_{\omega}^{2}+\Delta_{\chi}^{2}$, provided $\Delta_{\omega}$ and $\Delta_{\chi}$ are sufficiently small (no more than a few degrees).

\subsection{Relative intensities}

The relative intensities of the domains were calculated using the areas of the deconvoluted two-dimensional Gaussians that pass through the points of maximum intensity. Since the threedimensional domains exhibited a certain asymmetry, the two Gaussians oriented in directions $\omega$ and $\chi$ were considered for each domain. The areas were calculated according to the well known formula $A=2.5066 I_{\max } \beta$, where the maximum intensity $\left(I_{\max }\right)$ and the FWHM $(\beta)$ are given in the output of the program used for the deconvolutions. Finally, the arithmetic mean $A_{\mathrm{m}}$ of the two areas was calculated and used in the determination of the relative intensity $I_{\mathrm{r}}$ of the domains. Table 3 lists for each domain the areas $A_{\omega}$ and $A_{\chi}$, obtained for directions $\omega$ and $\chi$, respectively, the mean area $A_{\mathrm{m}}$, and the
Table 3

Relative intensities $\left(I_{\mathrm{r}}\right)$ of the domains in the aluminium crystal.

\begin{tabular}{lrrrr}
\hline Domain & \multicolumn{1}{c}{$A_{\omega}$} & \multicolumn{1}{c}{$A_{\chi}$} & \multicolumn{1}{c}{$A_{\mathrm{m}}$} & \multicolumn{1}{c}{$I_{\mathrm{r}}(\%)$} \\
\hline 1 & 3661 & 3722 & 3692 & 19.70 \\
2 & 6959 & 9952 & 8456 & 38.26 \\
3 & 20059 & 24147 & 22103 & 100.00 \\
4 & 15423 & 18684 & 17054 & 77.16 \\
5 & 1822 & 3356 & 2589 & 11.71 \\
\hline
\end{tabular}

relative intensity $I_{\mathrm{r}}$. It should be noted that domain No. 3 was taken as reference for the calculations.

\section{Concluding remarks}

In this work, a methodology for analysing the crystalline perfection of single crystals has been presented. The use of neutron diffraction enables the determination of the intrinsic crystalline domains of a crystal, therefore allowing an evaluation of its macroscopic crystalline perfection. Some of the procedures adopted during the data treatment and the determination of the characteristics of the domains are merely suggestions of practical ways to obtain the results and they should not be regarded as the only or best method to be used. Other solutions can be found, particularly for the determination of the FWHMs of domains and their relative intensities. Even the deconvolution of rocking curves is not absolutely necessary, unless the intrinsic characteristics of the domains are desired. Deconvolution is applied, or not, depending on the particular analysis and the results expected from the analysis.

It should be noted that, in this study, the aluminium crystal was larger than the neutron beam cross-sectional area and no scanning was performed during the measurements (see \$2). Taking into account the distance between the collimator exit and the sample $(35 \mathrm{~cm})$, the vertical and horizontal divergences, the beam dimensions, and the Bragg angle for reflection $111\left(\mathrm{ca} 14^{\circ}\right)$, we have calculated that approximately $60 \%$ of the crystal volume was immersed in the neutron beam during the measurements, but this has not impeded a good evaluation of the crystalline perfection of the crystal. Concerning its quality, it could be said that the aluminium crystal is not really a single crystal, although it was acquired as being one. The existence of five domains separated by angular distances ranging from 0.138 to $0.563^{\circ}$ (see Table 2) corroborates the above assertion. Use of such a crystal in a given application will depend on the particular needs of the application. It is important to note that the user of a crystal is the best judge if its quality is adequate, or not, for an intended use.

The authors are indebted to Dr Sonia L. Baldochi and Dr Izilda M. Ranieri, from the Crystal Growth Laboratory at IPEN-CNEN/SP, for providing the LiF single crystal used in this study. We are also grateful for financial support given by the International Atomic Energy Agency (IAEA, project No. 6974/R1/RB) and the Conselho Nacional de Desenvolvimento Científico e Tecnológico (CNPq) (contract No. 400397/93-5- 
FA). One of the authors (SM) acknowledges the grant of a fellowship by CNPq (contract No. 133150/94-1).

\section{References}

Arndt, U. W. \& Willis, B. T. M. (1966). Single Crystal Diffractometry. Cambridge University Press.

Baldochi, S. L., Mazzocchi, V. L., Parente, C. B. R. \& Morato, S. P. (1994). Mater. Res. Bull, 29, 1321-1331.

Hagenmuller, P. (1972). Editor. Preparative Methods in Solid State Chemistry. New York, London: Academic Press.
Mazzocchi, V. L. \& Parente, C. B. R. (1994). J. Appl. Cryst. 27, 475481.

Mazzocchi, V. L. \& Parente, C. B. R. (1998). J. Appl. Cryst. 31, 718725 .

Metairon, S. (1999). MSc dissertation, Universidade de São Paulo, São Paulo, SP, Brazil.

Press, W. H., Teukolsky, S. A., Vetterling, W. T. \& Flannery, B. P. (1994). Numerical Recipes in Fortran: The Art of Scientific Computing, 2nd ed. Cambridge University Press.

Rossmanith, E. (1986). Acta Cryst. A42, 344-348.

Spiegel, M. R. (1968). Mathematical Handbook of Formulas and Tables. Schaum's Outline Series. New York: McGraw-Hill Book Company. 